\title{
Techniques in plant telomere biology
}

\author{
Jiří Fajkus ${ }^{1,2}$, Eva Sýkorová1,2, and Andrew R. Leitch ${ }^{3}$ \\ BioTechniques 38:233-243 (February 2005)
}

\begin{abstract}
The role model systems have played in understanding telomere biology has been enormous, and understanding has rapidly transferred to human telomere research. Most work using model organisms to study telomerase and nontelomerase-based telomeremaintenance systems has centered on yeasts, ciliates, and insects. But it is now timely to put considerably more effort into plant models for a number of reasons: (i) the rice and Arabidopsis genome sequencing projects make data mining possible; (ii) extensive collections of insertion mutants of Arabidopsis thaliana enable phenotypic effects of protein gene knockouts to be analyzed, including for those genes involved in telomere structure, function (including, for example, in meiosis), and maintenance; and (iii) the variability of plant telomeres is considerable and ranges from the telomerase-mediated synthesis of the Arabidopsis-type (TT$T A G G G)$ and vertebrate-type (TTAGGG) repeats to sequences synthesized by telomerase-independent mechanism( $(s)$ that are still to be discovered. Here we describe how the understanding of telomere biology has been advanced by methods used to isolate telomeric sequences and prove that the putative sequences isolated are indeed telomeric. We show how assays designed to prove the activity of telomerase [e.g., telomeric repeat amplification protocol (TRAP)] lead not only to an understanding of telomere structure and function, but also to the understanding of cell activity in development and in the cell cycle. We review how assays designed to reveal protein/protein and protein/nucleic acid interactions promote understanding of the structure and activities of plant telomeres. Together, the data are making significant contributions to telomere biology in general and could have medical implications.
\end{abstract}

\section{INTRODUCTION}

The term "telomere" was first used in 1938 by H.J. Muller (1) who studied $\mathrm{X}$-ray-induced chromosome breaks in Drosophila. He noticed that irradiation-induced chromosome breaks caused chromosomes to join together while natural chromosome ends were protected against fusions and rearrangements. This functional definition of telomeres was substantially confirmed by studies of the breakage-fusionbridge cycle by $\mathrm{B}$. McClintock in maize in 1941 (2).

Despite the fact that plant studies have made important contributions from the outset of the telomere biology, many might perceive the study of plant telomeres as an auxiliary branch to the "main" topics, which offer attractive opportunities in human medicine, including diagnostics and rational treatment of cancer and the rejuvenation of mortal somatic cells and tissues for transplantation. However, the history of telomere research, indeed of all research, is littered with examples showing the importance of model systems in apparently peripheral fields. The study of plant telomeres remains restricted to only a few research groups in the world, but these groups have made some surprising discoveries, and the potential for plant telomere biologists is enormous. Furthermore, it is easy to envisage that plant telomere research could have a central role in medically related research because it is from plants that regulators and inhibitors of mammalian telomere activity are likely to be found.

The ciliate Tetrahymena $(3,4)$ proved a vital model system for our understanding of the molecular nature of telomeric DNA as well as the mechanism of its synthesis by telomerase. The searching for human telomere binding proteins has relied heavily on sequence homologies with previously characterized yeast or protozoan proteins. Today's knowledge of the diversity of telomere endmaintenance strategies, which includes telomerase and telomerase-independent mechanisms (e.g., recombination/ gene conversion), arose from studies on insects, yeasts, and plants (5-7), and only later similar processes were observed in human cells (e.g., recombination mediated end-maintenance in human tumor cells; Reference 8). Recent phylogenetic studies have shown that human-type telomeric sequences have replaced original Arabidopsis-type telomeres in a phylogenetically distinct clade of the monocotyledonous plant order Asparagales (9). This discovery has moved plant telomere biology closer to human biology than would be expected. Also within the Asparagales, in the Alliaceae family (the onions), is a telomerase-independent strategy of telomere maintenance. This evolutionarily derived condition makes these plants suitable model organisms for the study of alternative lengthening of telomeres (ALT) within a zero telomerase background.

Human telomere biology is focused on mechanisms of telomerase

${ }^{1}$ Academy of Sciences of the Czech Republic and ${ }^{2}$ Masaryk University Brno, Brno, Czech Republic, and ${ }^{3}$ Queen Mary University of London, London, UK 
regulation in cancer cells, but these cells are heavily altered by neoplastic transformation in comparison to normal cells. On the other hand, plant cells are naturally telomerase-competent (their telomerase activity is reversibly regulated in response to proliferation activity) and able to cope with mutations and stress conditions that are lethal for vertebrates. Totipotency of plant cells enables us to investigate changes in telomere metabolism during ontogenesis. It is time for a greater emphasis on plant models, particularly now when bioinformatic tools enable the fast transfer of knowledge between organisms. In particular, plant telomere research can take advantage of the libraries of Arabidopsis thaliana insertion mutants to evaluate phenotypic effects of any given mutation. These can be used to study mutational impacts at multiple levels from the molecule to the organism. Furthermore, combined mutations can be obtained through crossing. Thus, plant telomere research is timely. This review is designed to state our current knowledge of plant telomere research from a technical and practical perspective.

\section{Finding DNA Sequences at the Telomere}

The first characterization of plant telomeric DNA sequence was described in A. thaliana by Richards and Ausubel (10). They used an end-cloning strategy to construct a library enriched in telomeric DNA. Sequencing individual clones revealed that Arabidopsis telomeres were composed primarily of tandemly repeated blocks of $5^{\prime}$ TTTAGGG- ${ }^{\prime}$, with the rare occurrence of variant $5^{\prime}$-TTTAGAG-3' sequences. They noted that the length of individual telomeres was heterogeneous, with terminal restriction fragments (TRFs) ranging between 2.5 and $4 \mathrm{~kb}$. In the same work, the authors showed that homologous sequences are present in Zea mays telomeres and cross-hybridize to genomic DNAs of a number of other plant species. Considerably longer arrays of telomeric sequence (30-60 $\mathrm{kb})$ were then found in Solanum esculentum (tomato; Reference 11) and several other plant species including Hordeum vulgare (barley; Refer- ences 12 and 13), Oryza sativa (rice; References 14 and 15), and Nicotiana tabacum (tobacco; References 16 and 17). As with Arabidopsis telomeres, tomato telomeres contained a considerable quantity of variant repeats, namely $5^{\prime}$-TTAAGGG- ${ }^{\prime}$ sequences (11). These findings, as well as our recent results on telomere evolution in Asparagales (9), made it necessary to elucidate which of the sequence variants represent the true functional telomere and which reflect sequence degeneration at telomere-subtelomere junctions, occasional errors in synthesis by telomerase, or blocks of interstitial telomere repeats.

Experimental strategies to prove the occurrence of a particular telomeric sequence in an organism must, by necessity, involve a combination of several experimental techniques (Table 1). Recently, we successfully used slot-blot hybridization in combination with fluorescence in situ hybridization (FISH) and the telomeric repeat amplification protocol (TRAP) assay to define two major switch points in the evolution of Asparagales in which (i) the Arabidopsis-type of telomeric sequence was replaced by the humantype of telomeric sequence and (ii) the human-type of telomeric repeat was itself lost (9). Slot-blot hybridization of genomic DNA is especially useful for screening purposes and gives information at the DNA level about the presence of a probed sequence in the investigated genome. If a positive result is obtained by slot-blot hybridization, it remains necessary to demonstrate that the sequence is localized at the telomeres. Classically, this is done in two ways. The first approach uses BAL31 digestions of high molecular weight DNA. The enzyme progressively degrades DNA from double-stranded breaks. The degraded DNA is then digested with one or more restriction enzymes (which cannot cleave within telomeric repeats) and fragments separated by gel electrophoresis. After blotting, membranes are probed with either a terminal marker sequence or telomeric sequences. If the sequence is telomeric, then there should be a progressive shortening in lengths of TRFs with increasing BAL31 digestion time (10), and the TRF pattern at the zero BAL31 digestion time indicates the approximate telomere lengths. The second approach to confirm a sequence telomeric location is to use FISH or primed in situ labeling (PRINS).

Three different kinds of probes have been used for probing telomeres $(i)$ synthetic telomeric oligonucleotides; (ii) cloned fragments of telomeric DNA; and (iii) concatenated telomeric oligonucleotides (concatemers). These probes could be used for slot-blot or Southern hybridization as well as for in situ techniques. The cloned fragments of telomeric DNA and synthetic telomeric oligonucleotides have the advantage that the precise sequence being targeted is well defined $(9,12,17)$. The next advantage of synthetic oligonucleotides is the possibility to use them for obtaining strand-specific information, such as for the detection of overhangs of G-rich telomeric single-stranded DNA (18). The third kind of probeconcatemers-is prepared using PCR and synthetic oligonucleotides designed against both strands of the putative telomere sequence. These are concatenated into a complex probe with a great deal of heterogeneity in probe length (19) and, potentially, sequence heterogeneity. Despite this possible heterogeneity, these probes have been used to demonstrate the distribution of telomere sequences in a large range of plant species, especially by FISH $(20,21)$. In practice, any of these probes can be used because their hybridization patterns are very similar $(\mathrm{K}$. Neplechova, E. Sýkorová, and J. Fajkus, manuscript in preparation). However, concatemers are often rejected in favor of the first two probes mentioned. We cloned concatemers of seven different telomeric repeats (Homo TTAGGG, Arabidopsis TTTAGGG, Chlamydomonas TTTTAGGG, Bombyx TTAGG, Oxytricha TTTTGGGG, Tetrahymena TTGGGG, and Ascaris TTAGGC), and we found the error ratio in products to be $10^{-3}$, which is consistent with the observation of Sahara et al. (22). Concerns have also been raised over the specificity of probes derived from very similar telomeric repeats, leading to possible cross-hybridization. Difference between telomeric repeat units is generally only a singlenucleotide mismatch or insertion/ 
deletion. Our results indicated that only synthetic oligonucleotides derived from the Arabidopsis-type telomere cross-hybridized with the Chlamydomonas-type of telomeric repeat, but not vice versa and only when washed under low stringency. The concatemers, cloned telomeric fragments, and all the other synthetic oligonucleotide probes showed negligible levels of cross-hybridization in any combination investigated. Thus, the positive signal obtained with multiple probes should not be interpreted as a result of the cross-hybridization.

PRINS is particularly amenable for telomeric localization experiments because telomeric minisatellite sequences usually contain just two or three of the four possible nucleotides in each DNA strand (e.g., 5'-TTTAGGG$3^{\prime}$ in plants). Thus, the dideoxy-PRINS variant of the technique can be used (23). Here DNA polymerase I extends one of the DNA strands. The nucleotide that is not found in the extended strand is provided in the dideoxyform (ddNTP), which terminates the PRINS reaction if it gets incorporated. Therefore, any hybridization of the telomeric primer to nontelomeric sites will not be extended, and the reaction will not label this DNA.

The basic disadvantage of FISH to mitotic chromosomes is its low resolution $(2-10 \mathrm{Mb})$. The improvement of resolution can be achieved using FISH to spread pachytene chromosomes, which are 20-25 times longer (24). Still better resolution can be achieved using extended DNA fibers (EDF) prepared from interphase nuclei (25). This technique has a stretching ratio of $2-3 \mathrm{~kb}$ per micrometer and a spatial resolution of less than $1 \mathrm{~kb}$. However, the structural landmarks of chromosomes are absent in this type of preparation. Therefore, probes from adjacent (subtelomeric) regions are necessary for the proper interpretation of results, and it is useful to combine extended DNA fiber fluorescent in situ hybridization (EDF-FISH) with FISH to pachytene or mitotic chromosomes (Table 1). These approaches have been successful in characterizing arrangements of variant telomeric repeats and associated subtelomeric repeats in chromosomes of tomato (26), white campion (Silene latifolia; Reference 27), and Ornithogalum virens (28). Because the resolution of EDF-FISH falls within the range of PCR, it is possible to amplify particular sequence variants within the telomere array or at telomere-subtelomere junction regions to obtain DNA sequence (27). Alternatively, EDF-FISH may be used to verify previous results derived from the sequence analysis of PCR products (29-32). Recently, a novel high-resolution FISH strategy was described that uses super-stretched flow-sorted plant chromosomes as targets (33). The technique allows the longitudinal extension of chromosomes to more than 100 times their original metaphase size. The protocol involves flow cytometric sorting of metaphase chromosomes, mild proteinase $\mathrm{K}$ digestion of air-dried chromosomes on microscopic slides, and stretching with ethanol:acetic acid (ratio of 3:1). Spatial resolution of neighboring loci is as high as $70 \mathrm{~kb}$. Stretched chromosomes have advantages over EDF from interphase nuclei because they retain chromosomal integrity. Although the method is confined to species for which chromosome flow sorting has been developed, it provides a unique system for controlling the degree of stretching of mitotic chromosomes and can be used to generate, using FISH, highresolution bar-coded chromosomes.

An interesting variant of FISH is chromatid orientation FISH (COFISH), which can be used to determine if tandem sequences, including those at telomeres, are arranged head-to-tail or whether orientations are mixed (34). In this procedure, cells are grown in the presence of $\mathrm{BrdU}$ for one round of replication so that sister chromatids are singly substituted. After culture, routine chromosome preparations are exposed to ultraviolet (UV) light and exonuclease III treatment to remove the newly synthesized strands. At the end of the procedure, chromatids are singlestranded (i.e., contain only a complementary prereplication DNA strand). Making use of a single-stranded oligonucleotide probe, only one chromatid will show a hybridization signal if the tandem repeats are oriented headto-tail along the DNA strand. This technique is particularly useful for studies of recombination events in telomeres. In some telomerase-negative tumors, telomeres become lengthened by ALT, a recombination-mediated DNA replication process in which telomeres use other telomeric DNA as a copy template. Using CO-FISH, it has been found that postreplicative exchange events involving a telomere and another TTAGGG repeat tract occur at remarkably high frequencies in ALT cells and rarely or never in nonALT cells (35). These exchanges are telomere specific because there was no correlation with sister chromatid exchange rates at interstitial locations. The technique has not yet been applied to plant chromosomes as far as we know, but, clearly, it has promising application for plant telomere researchers (e.g., to study telomere metabolism in plant species lacking telomerase activity). Finally, the peptide nucleic acid (PNA) probes, used routinely in human in situ techniques (36), have been recently used to demonstrate that Aloe and Othocalis carry the vertebrate-type of telomere sequence $(37,38)$.

Demonstrating the terminal position of candidate DNA sequence by both BAL31 digestion and FISH is usually considered sufficient to prove its telomeric location. However, there may still be doubts arising as to the sequence functional significance (e.g., recently, it has been shown that there are multiple variants of minisatellite telomeric sequence in the order Asparagales; Reference 9). FISH is not able to distinguish between telomeric and subtelomeric signals. Likewise, BAL31 digestion analysis of TRFs can hardly distinguish terminal sequences from those at telomere/subtelomere boundaries. The distinction might be significant because only the most distal sequences are actually telomeres and are maintained by telomerase. Perhaps the best evidence for terminal, telomeric sequences comes from in vitro elongation of substrate primers in the telomerase extract of a given plant (e.g., plant TRAP assay), and then sequence analysis of the products is obtained. The only problem is that the conditions of the telomerase reaction might influence its activity (e.g., fidelity of synthesis and processivity), and these will not exactly correspond 
Table 1. Techniques Used in Analysis of Telomeric DNA

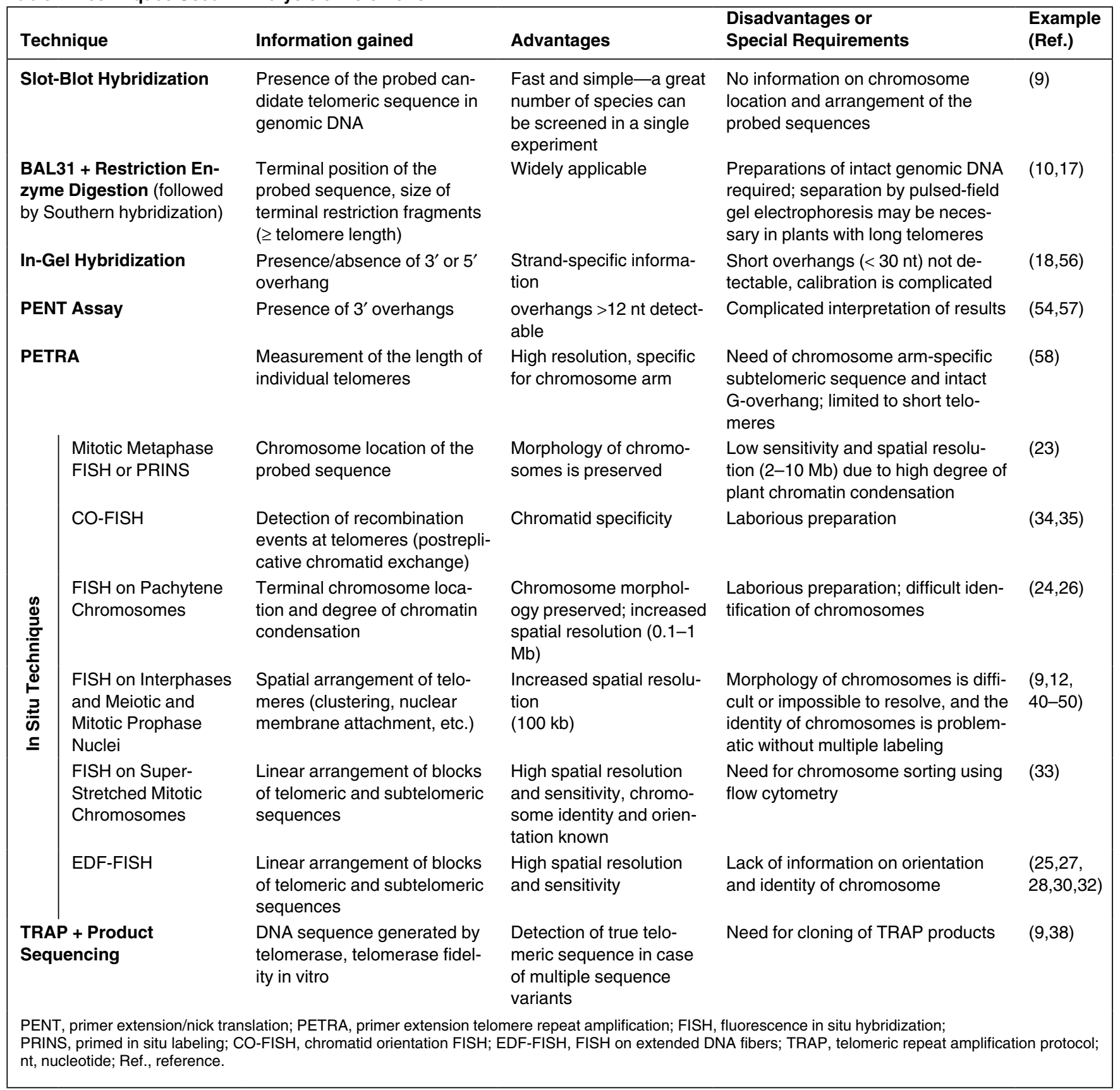

to those in vivo.

\section{Using FISH to Analyze Telomere Distributions in Nuclei}

FISH has been used extensively to localize telomere positions in interphase and meiotic cells of plants. Rabl (39) observed that chromosomes decondense at telophase in a pattern resembling the anaphase organization, with centromeres at one pole and trailing telomeres at the other. Then, at the subsequent prophase, the chromosomes condensed once again in the same orientation, indicating their organization had remained unaltered through interphase. This Rabl configuration of chromosomes at interphase was confirmed using FISH and labeled oligonucleotide probes against Arabidopsis-type telomeres to $H$. vulgare meristematic cells. Here it was shown that telomeres are indeed clustered at one pole (12). This pattern of chromosomes and distribution of telomeres have since been observed in dividing cells of many, but not all, plant and animal species. Possibly species with small genomes and/or chromosomes lack the Rabl organization at interphase $(40,41)$. This may be because the pattern is lost in these species as a consequence of small chromosome movements at interphase (42).

Fluorochrome-labeled oligonucle- 
otides designed against the Arabidopsis-type telomeres have also been used extensively to demonstrate the role of telomeres in plant meioses particularly in Z. mays (maize) and Secale cereale (rye). Whole mounts of meiotic tissue and meiotic cells in prophase I have been labeled by FISH, and in three-dimensional (3-D) reconstructions, the distributions of telomeres have been analyzed through meiosis. The earliest stages of prophase I are characterized by the formation of the bouquet arrangement of the chromosomes where the telomeres first attach to the nuclear envelope at one pole and then move together into tight clusters at the leptotene/zygotene boundary $(40,43)$. This organization is an almost universal feature of eukaryotes (44) and is driven by interactions with the inner membrane of the nuclear envelope (45). The clustering of telomeres after their attachment to the nuclear envelope is inhibited by colchicine, an inhibitor of microtubule polymerization. But not all drugs that inhibit microtubule formation have this effect (46). Thus, the precise role of colchicine in inhibiting telomere clustering is unclear, particularly as immunolocalization reveals an abundance of microtubules at the opposite pole to the telomeric pole of the bouquet (47).

In maize, there are mutants with aberrant meiosis and impaired fertility. Some of these mutants also have abnormal telomere distribution
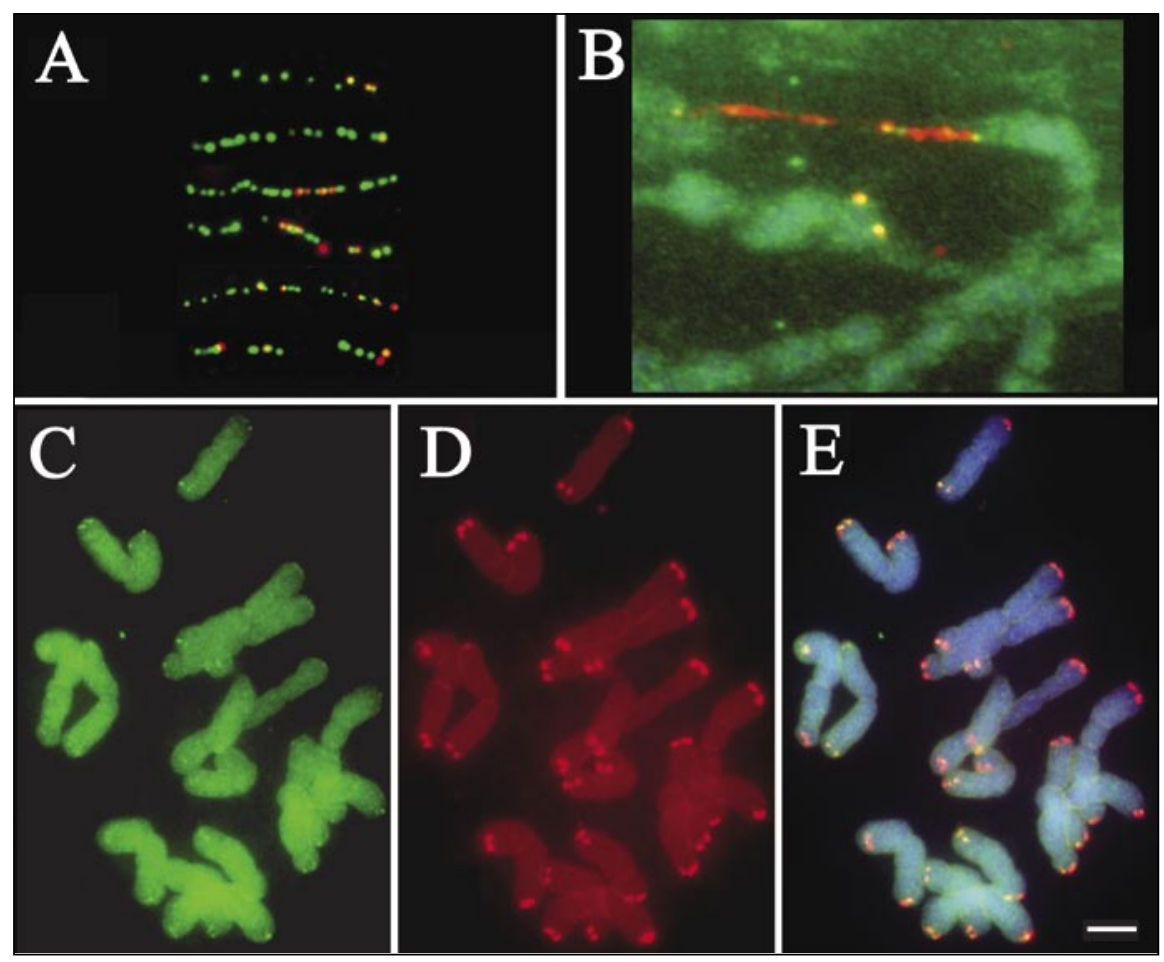

Figure 1. Analysis of plant telomeres in situ. (A) Extended DNA fiber fluorescent in situ hybridization (EDF-FISH) to Ornithogalum virens (monocotyledonous plant in order Asparagales) probed with concatenated Arabidopsis-type telomere sequence TTTAGGG labeled with biotin and detected with avidin$\mathrm{Cy}^{\mathrm{TM}} 3$ (red fluorescence) and also with concatenated human-type telomere sequence TTAGGG labeled with digoxigenin (DIG) and detected with anti-DIG-fluorescein isothiocyanate (FITC; green fluorescence). Note that most label is to the human-type of motif, although Arabidopisis-type motifs are found interspersed and at terminal positions of extended DNA fibers (EDF; Reference 28). (B-E) Metaphases of Ornithogalum umbellatum (GenBank ${ }^{\circledR}$ accession Kew 82.919, Asparagales) probed with concatenated Arabidopsis-type telomere sequence labeled with DIG and detected with anti-DIG-FITC (green fluorescence) and also with concatenated human-type telomere sequence labeled with biotin and detected with avidin-Cy3 (red fluorescence). (B) Both labels superimposed, and the chromatin is counterstained blue with DAPI. Note that, similar to the EDF-FISH in panel A, there is only a small amount of Arabidopsistype telomere sequences. The extension of the telomere fiber from the chromatid is a useful property for these analyses and can be an artifact generated by the drug used to accumulate metaphases [saturated aqueous solution of hexachlorocyclohexane (Sigma, St. Louis, MO, USA) for $4 \mathrm{~h}$ ]. (C-E) Metaphase with each label separated by color. Note the small amount of Arabidopsis-type sequence visible at the termini of only some chromosome arms. Scale bar $=10 \mu \mathrm{m}$. in meiosis, and their analyses have shed considerable light on the roles of telomeres in meiosis and the mechanism of meiosis in general. The paml (plural abnormalities of meiosis) gene is associated with a lack of telomere clustering in the formation of the bouquet, the phenotype being reminiscent of treatment with colchicine (48). Telomere clustering itself is probably involved with homologue searching (44), although the gene phs 1 is needed for appropriate homologue synapsis in maize (49). Likewise, mutants of the gene dys 1 (desynaptic1) result in an incomplete bouquet with telomeres failing to cluster properly and $d y$ (desynaptic) results in the early release of telomeres from the nuclear envelope at pachytene (50).

\section{The Packing of DNA at the Telomere}

Most DNA at telomeres is packed with histones as nucleosomal DNA. However, in micrococcal nuclease digestions, telomeric chromatin shows unusually short nucleosome spacing and extensive subnucleosomal fragmentation of mononucleosomeand dinucleosome-sized particles. This arises as a consequence of nucleosome sliding on the telomeric chromatin fiber in the absence of nucleosome positioning signals $(17,51)$. On the other hand, nucleosomes in long fibers of telomeric chromatin apparently do not slide. Because the homogeneous tracts of simple telomere sequences seem not to carry any nucleosome positioning signal, the regularity of nucleosome repeat size must be dictated somehow by the chromatin structure itself. A possible explanation is that the telomeric DNA is packed as described in the columnar packing model (52). In this model, telomeric DNA is continuously wound around columns of stacked histone octamers. Linkers are deformed in the same manner as for the deformable part of nucleosome DNA, and octamer-to-octamer stacking contacts cooperatively maintain the overall structure and prevent the whole nucleosomal array from sliding. This packing structure potentially expands into telomere-associated subtelomeric regions $(31,53)$.

Telomeric DNA also has distinctive 
nucleoprotein structures that have functional roles. The $3^{\prime}$ overhang of a G-rich telomeric strand is of particular interest because it is folded to form telomere loops. These overhangs and also possibly telomeric loops may be conserved across plant taxa, including plants with human-type telomeres. Evidence for this comes from the analysis of the telomeres of several species (e.g., Hordeum, Iris, Arabidopis; References 28 and 54) and from electron microscopy to the garden pea (Pisum sativum; Reference 55). Singlestranded overhangs can be detected using in-gel hybridization (56), a method in which DNA samples are separated on native agarose gels and then hybridized with C- and G-strandspecific probes. The negative control for this experiment can be conducted using nucleases that remove singlestranded overhangs (e.g., exonuclease 1 to remove $3^{\prime}$ overhangs), and positive controls can be conducted using exonuclease treatment of cloned substrates (T7 gene 6 exonuclease generates $3^{\prime}$ overhangs, exonuclease III generates $5^{\prime}$ overhangs). A further control is to reprobe the gel after alkali denaturation. These approaches have been used to demonstrate that G-overhang lengths increase in A. thaliana Ku70 mutants (18). Alternatively, G-overhangs can be detected using the primer extension/ nick translation (PENT) assay (57). In this assay, a C-strand telomeric primer is annealed to the telomeric G-overhang and the primer extended using DNA PolI or TaqI DNA polymerase in the absence of dGTP (to stop the extension at telomere-subtelomere junction). The newly synthesized strand $\left(\mathrm{C}_{\mathrm{s}}\right)$ replaces the original telomeric strand $\left(\mathrm{C}_{\mathrm{o}}\right)$ because of the $3^{\prime}$ exonuclease activity of DNA polymerases. The reaction leaves a nick between $\mathrm{C}_{\mathrm{s}}$ and $\mathrm{C}_{\mathrm{o}} \cdot \mathrm{C}_{\mathrm{s}}$ strands are then separated from bulk DNA by alkaline electrophoresis, and their lengths are measured after hybridization with the telomeric repeat probe. The technique was the first to demonstrate G-strand overhangs in plant chromosomes (54).

The presence of telomeric Goverhangs is utilized in a technique designed to measure individual telomere lengths in plants with relatively short telomeres and known subtelomeric sequences. The method is a PCR approach called primer extension telomere repeat amplification (PETRA; Reference 58) and is a development of the single telomere length analysis (STELA) method previously described in human chromosomes (59). In PETRA, an adaptor primer (which consists of $3^{\prime}$ telomeric part and $5^{\prime}$ partbearing nontelomeric tag) is annealed to the telomeric G-overhang, and the sequence is extended using DNA PolI. Then, a primer specific for a known subtelomeric sequence specific to a particular chromosome arm is used with a primer identical to the tag of the adaptor primer in a PCR. The amplified products are then detected by Southern hybridization using a telomeric repeat probe.

\section{Analysis of Plant Telomerase}

Telomerase-mediated DNA synthesis is the most common mechanism of telomere elongation in plants. Plant telomerase activity was first detected in a tobacco BY-2 cell culture in a direct assay that used a Gstrand telomeric oligonucleotide as a substrate for telomerase activity (60). Concurrently, TRAP for plants was developed and used to demonstrate telomerase activity in extracts from meristematic tissues of $A$. thaliana, barley, carrot, cauliflower, soybean, and rice $(61,62)$. Important modifications of this protocol for plants (62) with respect to the human TRAP assay (63) include: (i) using a different extraction buffer; (ii) enriching the extract with telomerase by precipitating the crude telomerase extract with polyethylene glycol (PEG) 8000, resuspending the pellet in a quarter of the original volume of the extraction buffer before centrifuging and collecting the supernatant; and (iii) performing the telomerase extension step at room temperature or $26^{\circ} \mathrm{C}(64)$. Further optimization of the assay now means that radioisotopic detection of the reaction products can be replaced by fluorescence detection after SYBR ${ }^{\circledR}$ Green staining $(64,65)$. The sensitivity of the technique is sufficient to reproducibly detect telomerase activity in extracts of meristematic tissues containing as little as $1 \mathrm{ng}$ of total protein. A low protein concentration in telomerase assays decreases the risk of false-negative results caused by inhibitors of the polymerase performing the amplification step of the TRAP assay.

The gene coding for the telomerase catalytic subunit in Arabidopsis (AtTERT) was found from predicted homology to conserved motifs in the TERT subunits of human, protozoa, and yeast $(66,67)$. The abundance of AtTERT transcripts correlated with telomerase activity. An Arabidopsis line with a T-DNA insertion disrupting the activity of the AtTERT gene was identified, and AtTERT-null plants were prepared by self-pollination of a heterozygous plant. PCR analyses and TRAP assays on floral buds from the knockout lines confirmed that AtTERT is a single-copy gene essential for telomerase activity. The length of TRFs revealed that the null mutants have progressive telomere shortening of 250 to 500 bp per generation (67), which is in contrast to stable telomere lengths in wild-type plants (65). The telomerasenull plants become dysfunctional in the sixth generation, as demonstrated by increased frequency of anaphase bridges, morphological changes in vegetative and reproductive organs, and decreased efficiency of seed germination. Mutants ultimately had arrested growth at a terminal vegetative state in the 10th generation. Although some effects of telomere dysfunction resemble those observed in telomerasenull animals, the plants did show differences in their response to telomere dysfunction. Aberrations in cell proliferation in late-generation mutant plants were partially compensated for by cell enlargement. In addition, the leaves of these plants remained green and metabolically active for longer periods of time than wild-type plants and showed no signs of senescence or apoptosis (68).

Although much is now known about the catalytic subunit of plant TERT from sequence analysis, the RNA subunit of plant telomerase, TR, remains an enigma. The conserved telomerase motifs of the catalytic subunit were relatively easy to characterize by either database mining $(66,67,69)$ or reverse genetics using mRNA isolated from tissues with telomerase activity (E. Sýkorová, A. Leitch, and J. Fajkus, 
manuscript in preparation). However, there is poor sequence conservation of the RNA subunits even among closely related species. Conventional molecular biology techniques used to isolate the RNA subunit in other eukaryotes have failed to be successful in plants. It might be expected that the low-stringency hybridization techniques used to isolate protozoa telomerase RNA would fail because, in protozoa, advantage is taken of the considerable telomerase activity involved in capping of the approximately $10^{4}$ genome fragments in each macronucleus. But it is not clear why the approaches used to obtain human TR have also failed to work in plants. The approach used to isolate human telomerase RNA included the preparation of a PCR-cDNA library enriched in telomerase RNA sequences either by subtractive hybridization with a PCR-cDNA library from telomerasenegative tissues or by isolating RNA from partially purified telomerase extracts of strongly telomerase-positive tissues or cell cultures. Then cDNA fragments were hybridized to a biotinylated 12-nucleotide complement of the predicted template sequence and purified using streptavidin magnetic beads. The selection process was repeated 3 or 4 times, and resulting cDNA fragments were cloned and sequenced. The application of bioinformatics to identify plant TR may prove successful, but it will certainly require more sophisticated algorithms than simple sequence alignments, and it will probably require advantage to be taken of conserved structural features (e.g., the so-called "pseudoknot").

\section{The Regulation of Plant Telomerase}

TRAP assays are now used with analyses of telomere lengths (evaluated from TRFs) to study the developmental controls of telomerase activity. In one such study (64), we analyzed telomerase activity and telomere lengths in tobacco leaves, in callus cultures initiated from leaf tissues of the same plants, and leaves of plants regenerated from these calli. The process was then repeated using the regenerants as the source material. The process of dedifferentiation or differentiation of plant cells can be controlled by the ratio of hormones NAA (1-naphtylacetic acid) and BAP (6-benzylaminopurine). Results showed that telomerase activity is hardly detectable in the original leaves, is up-regulated in proliferating callus cultures, and falls again in leaves of regenerated plants. This pattern was repeated in the second round of culturing and regeneration. Telomere lengths remained unchanged in both generations of calli as well as in leaves of all three somatic plant generations despite dramatic changes in cell division rates and corresponding variations in telomerase activity. This demonstrates a reversible and precise regulation of telomerase activity compensating for the replicative loss of telomeric DNA with each cell cycle. This telomerase-competent status of plant cells is probably a necessary condition for their totipotency. Similar results were observed in $S$. latifolia, which has significantly shorter telomeres (65).

Reverse transcription PCR (RTPCR) experiments suggest that the primary level of regulation of telomerase activity is the transcription of its catalytic subunit TERT. In $O$. sativa, tissue-specific splicing of TERT RNA has been observed (69), suggesting a role for splicing in regulation that is similar to that described in human TERT (70,71). Cell cycle-dependent regulation has been observed in synchronized tobacco BY-2 cells $(72,73)$, where telomerase activity peaked at early $S$ phase of the cell cycle. Interestingly, blocking S-phase progression with aphidicolin (inhibitor of DNA polymerase $\alpha$ ) or hydroxyurea (inhibitor of ribonucleotide reductase) did not influence telomerase activity. However, exposure of cells to olomoucine, an inhibitor of the Cdc2/Cdk2 cyclin-dependent kinases, blocking transition from $\mathrm{G} 1$ to $\mathrm{S}$ phase, almost completely inhibited the induction of telomerase activity. The plant hormone auxin, which induces cell proliferation and is thought to be required for $\mathrm{G} 1$ to $\mathrm{S}$ phase transition and the initiation of DNA replication, elevated by 2- to 3-fold telomerase activity at early $S$ phase in a dosedependent manner. Another phytohormone, abscisic acid, which induces the cyclin-dependent protein kinase inhibitor, effectively abolished both the auxin- and S-phase-specific activation of telomerase in a concentration- and time-dependent fashion. Treatment of synchronized BY-2 cells with the protein kinase inhibitor staurosporine or H-7 prevented the S-phase-specific activation of telomerase activity. When okadaic acid or cantharidin (inhibitors of protein phosphatase 2A, PP2A) was applied to the cells, the S-phase-specific high level of telomerase activity was continuously maintained in the cell cycle for at least $14 \mathrm{~h}$ after release from M-phase arrest. Exogenous PP2A rapidly abrogated telomerase activity in vitro while okadaic acid and cantharidin blocked the action of PP2A, effectively restoring in vitro telomerase activity. Thus, the cell cycle-dependent control is mediated in plants by antagonistic functions of phytohormones, auxin, and abscisic acid. Furthermore, the data show that the regulation of plant telomerase involves reversible protein phosphorylation and dephosphorylation, as also occurs for human TERT (74).

\section{The Role of Telomerase in Chromosome Healing}

Using a biochemical approach, it has been found that telomerases from different plant species differ in their ability to elongate nontelomeric templates and in sequence fidelity of the de novo synthesized telomeres (75). Sequence analysis of TRAP assay products using a set of nontelomeric primers ending with a different nucleotide or dinucleotide has shown that some telomerases (e.g., soybean) lack the ability to elongate primers not ending in telomeric nucleotides. On the other hand, Arabidopsis and maize telomerases efficiently extended completely nontelomeric primers by positioning the $3^{\prime}$ terminus at a preferred site on the RNA template. In contrast, $S$. latifolia and sorghum telomerases annealed nontelomeric templates at alternative sites and produced several-fold higher frequency of errors. These results reflect highly variable enzyme-DNA interactions in vitro with implications for the process of "chromosome healing." Perhaps chromosome healing needs to be considered in a much broader 
context than just the de novo addition of telomeres to chromosome breaks. When chromosome fragmentation was induced in tobacco BY-2 cell culture by cadmium sulfate, chromosomes were fragmented into $50-200 \mathrm{~kb}$ pieces on the third day of incubation. Surprisingly, fragmentation could be reversed 2 days after cadmium withdrawal. Late stages of fragmentation and a complete recovery of cell viability were accompanied by a 2.5 -fold increase in telomerase activity, suggesting its participation in a remarkably efficient DNA repair process (76). The pathway of plant telomerase activation by large-scale chromosome fragmentation remains unknown, but this study certainly demonstrated the enormous tolerance of plant cells to genotoxic stress and to uncapped chromosome breaks.

\section{Proteins Complexed with Plant Telomeres}

Telomeric proteins are important for chromatin folding, capping, and end maintenance. Research progress on specific telomeric proteins in plants lags with respect to the commonly used model systems. Nevertheless, understanding has been derived by the detailed functional characterization of Arabidopsis orthologs of repair proteins Ku70 (77,78), Ku80 (79), Mre11 (80), and Rad50 (81) involved in telomere metabolism. Arabidopsis T-insertion mutants are a valuable resource to characterize phenotypic, cytogenetic, and molecular effects of a protein's absence. Ríha and Shippen (18) showed that double mutants deficient in both Ku70 and TERT showed accelerated telomere shortening despite the extension of the single-stranded G-overhangs. Several candidate plant telomere binding proteins, forming specific complexes with either single-stranded or double-stranded telomeric DNA, have been identified by electrophoretic mobility shift or by database searches, but, apart from a few examples, little is known about their functions $(82,83)$. An exception is a $40-\mathrm{kDa}$ protein identified in nuclear extracts of telomerase-negative tissues (leaves) of $N$. tabacum and S. latifolia (84). This protein forms salt-stable complexes with the G-rich telomeric strand ([TTTAGGG $]_{\mathrm{n}}$ ) and causes species-nonspecific inhibition of plant telomerase owing to a reduced accessibility of the G-overhang. Recently, an $18-\mathrm{kDa}$ telomeric G-strand binding protein containing two RNA binding domains was shown to have an inhibitory effect on telomerase activity in A. thaliana (85). Another G-strandbinding protein $(28 \mathrm{kDa})$, with two putative RNA binding domains, was found in the tobacco BY-2 cell line (86), but any inhibitory effect on telomerase activity remains untested. It is likely that proteins binding to G-overhangs will be of high functional importance because these chromatin structures are essential for telomere capping and for telomere elongation by telomerase.

The study of DNA binding proteins should be followed by searches for their binding partners (i.e., proteins that are recruited to telomeres by proteinprotein interactions). Some telomeric proteins show both DNA binding and protein-protein interactions. An example is human POT1 protein, which binds either single-stranded telomeric G-strand overhang or can be recruited to the double-stranded part of the telomere via TRF1 protein complex (87). When bound to the single-stranded telomeric G-strand overhang, it functions to protect the overhang from degradation and influences accessibility to telomerase (88). When it is recruited to double-stranded DNA, it interacts with PTOP protein, which is bound to TIN2, a protein in turn bound to TRF1, which is attached to DNA (89). In this way, human POT1 protein functions as a terminal transducer of telomere length control. To find out whether analogous regulation pathways exist in plants, it is necessary to determine which of the candidate telomere binding myb-like proteins $(90,91)$ is indeed a functional analogue of TRF1 and whether any of the two Arabidopsis orthologs of Pot1 acts in a way similar to the human Pot1 protein (92). For these studies, general approaches such as two-hybrid assay or immunoprecipitation techniques should be combined with the use of Arabidopsis mutants.

Histones are the most abundant proteins at the telomere and, despite the presence of specific telomere binding proteins, most of the telomere is folded into nucleosomal DNA in plants (17) as occurs in mammals $(93,94)$. The organization of the histones may however be different at telomeres, as described in the columnar model of telomeric DNA packing. There may also be specific variants of core or linker histones (H1types) and/or histone modifications present in telomeric chromatin (95).

Recently, it has been shown that heterochromatin protein 1 (HP1) interferes with interactions between human TERT and the telomere (96). The HP1like protein from fission yeast (Swi6) is directly involved in the formation of heterochromatin structure at the telomere and is apparently recruited to telomeres by the Taz1 telomere-repeat binding protein (97). Mutant mice, which are null with respect to histone methyltransferases Suv39h1 and Suv39h2 (which govern methylation of histone H3-Lys9 in heterochromatic regions), show abnormal elongation of telomeres and reduced binding of HP1-like chromobox proteins. These results indicate epigenetic regulation of telomere length. It remains to be shown whether this level of regulation also exists in plant cells, which are naturally telomerase-competent. Again, Arabidopsis mutants can hopefully be used to solve this question.

\section{CONCLUSION}

There is now an expanding range of techniques to analyze telomere structure, function, and activity. These include TRAP, telomere folding (MNase digestion), telomere organization (FISH and PRINS), protein/ protein (two hybrid screens), and protein/nucleic acid (gel retardation assays) interactions, and sophisticated cloning strategies to isolate telomere and telomere-associated sequences. These methods can all be used with the powerful resource provided by Arabidopsis mutants deficient in any prescribed protein gene. The complexity of protein-protein and protein-nucleic acid interactions at telomeres is likely to be an area of considerable challenge over the upcoming years, and the networks revealed are likely to prove very fruitful. Telomere research on 
plants can reveal similarities and differences with other model systems. It can also be used to exploit the astonishing diversity of telomere maintenance strategies found. Thus, plant telomere research promises to provide us with an invaluable impetus to a general understanding of telomere biology.

\section{ACKNOWLEDGMENTS}

We thank Marie Skleničková and Kar Yoong Lim for providing fluorescence microscopy images. Our research is supported by the Grant Agency of the Czech Republic (projects 204/02/0027 and 521/05/0055 to J.F., 204/04/P104 to E.S.), Leverhulme Trust to A.R.L., and the institutional support to J.F. and E.S. (MSM143100008, MSM0021622415, and AVOZ50040507).

\section{COMPETING INTERESTS STATEMENT}

The authors declare no competing interests.

\section{REFERENCES}

1.Muller, H.J. 1938. The remaking of chromosomes. Collecting Net. 13:182-198.

2.McClintock, B. 1941. The stability of broken ends of chromosomes in Zea mays. Genetics 26:234-282.

3.Blackburn, E.H. and J.G. Gall. 1978. A tandemly repeated sequence at the termini of the extrachromosomal ribosomal RNA genes in Tetrahymena. J. Mol. Biol. 120:33-53.

4.Greider, C.W. and E.H. Blackburn. 1985. Identification of a specific telomere terminal transferase activity in Tetrahymena extracts. Cell 43:405-413.

5.Biessmann, H., J.M. Mason, K. Ferry, M. d'Hulst, K. Valgeirsdottir, K.L. Traverse, and M.L. Pardue. 1990. Addition of telomere-associated HeT DNA sequences "heals" broken chromosome ends in Drosophila. Cell 61:663-673.

6.Lundblad, V. and E.H. Blackburn. 1993. An alternative pathway for yeast telomere maintenance rescues est1-senescence. Cell 73:347360 .

7.Fuchs, J., A. Brandes, and I. Schubert. 1995. Telomere sequence localization and karyotype evolution in higher plants. Plant Syst. Evol. 196:227-241.

8.Bryan, T.M., A. Englezou, L. Dalla-Pozza, M.A. Dunham, and R.R. Reddel. 1997. Evidence for an alternative mechanism for maintaining telomere length in human tumors and tumor-derived cell lines. Nat. Med. 3:12711274.
9.Sýkorová, E., K.Y. Lim, Z. Kunická, M.W. Chase, M.D. Bennett, J. Fajkus, and A.R. Leitch. 2003. Telomere variability in the monocotyledonous plant order Asparagales. Proc. R. Soc. Lond. B. Biol. Sci. 270:18931904.

10.Richards, E.J. and F.M. Ausubel. 1988. Isolation of a higher eukaryotic telomere from Arabidopsis thaliana. Cell 53:127-136.

11.Ganal, M.W., N.L. Lapitan, and S.D. Tanksley. 1991. Macrostructure of the tomato telomeres. Plant Cell 3:87-94.

12.Schwarzacher, T. and J.S. Heslop-Harrison. 1991. In situ hybridization to plant telomeres using synthetic oligomeres. Genome 34:317323.

13.Roder, M.S., N.L. Lapitan, M.E. Sorrells, and S.D. Tanksley. 1993. Genetic and physical mapping of barley telomeres. Mol. Gen. Genet. 238:294-303.

14.Wu, K.S. and S.D. Tanksley. 1993. PFGE analysis of the rice genome: estimation of fragment sizes, organization of repetitive sequences and relationships between genetic and physical distances. Plant Mol. Biol. 23:243-254

15.Wu, K.S. and S.D. Tanksley. 1993. Genetic and physical mapping of telomeres and macrosatellites of rice. Plant Mol. Biol. 22:861872.

16.Suzuki, K., Y. Yamagiwa, T. Matsui, and K. Yoshida. 1994. Restriction enzyme-resistant high molecular weight telomeric DNA fragments in tobacco. DNA Res. 1:129-138.

17.Fajkus, J., A. Kovařík, R. Královics, and M. Bezděk. 1995. Organization of telomeric and subtelomeric chromatin in the higher plant Nicotiana tabacum. Mol. Gen. Genet. 247:633-638.

18. Ríha, K. and D.E. Shippen. 2003. Ku is required for telomeric C-rich strand maintenance but not for end-to-end chromosome fusions in Arabidopsis. Proc. Natl. Acad. Sci. USA 100:611-615.

19.Ijdo, J.W., R.A. Wells, A. Baldini, and S.T. Reeders. 1991. Improved telomere detection using a telomere repeat probe (TTAGGG)n generated by PCR. Nucleic Acids Res. 19:4780.

20.Cox, A.V., S.T. Bennett, A.S. Parokonny, A. Kenton, M.A. Callimassia, and M.D. Bennett. 1993. Comparison of plant telomere locations using a PCR-generated synthetic probe. Ann. Bot. 72:239-247.

21.Adams, S.P., T.P. Hartman, K.Y. Lim, M.W. Chase, M.D. Bennett, I.J. Leitch, and A.R. Leitch. 2001. Loss and recovery of Arabidopsis-type telomere repeat sequences $5^{\prime}$ (TTTAGGG)(n)-3' in the evolution of a major radiation of flowering plants. Proc. R. Soc. Lond. B. Biol. Sci. 268:1541-1546.

22.Sahara, K., F. Marec, and W. Traut. 1999. TTAGG telomeric repeats in chromosomes of some insects and other arthropods. Chromosome Res. 7:449-460.

23.Krejčí, K. and J. Koch. 1999. An in situ study of variant telomeric repeats in human chromosomes. Genomics 58:202-206.

24.Fransz, P., S. Armstrong, C. Alonso-Blanco, T.C. Fischer, R.A. Torres-Ruiz, and G. Jones. 1998. Cytogenetics for the model system Arabidopsis thaliana. Plant J. 13:867-
876.

25.Fransz, P.F., C. Alonso-Blanco, T.B. Liharska, A.J. Peeters, P. Zabel, and J.H. de Jong. 1996. High-resolution physical mapping in Arabidopsis thaliana and tomato by fluorescence in situ hybridization to extended DNA fibres. Plant J. 9:421-430.

26.Zhong, X.B., P.F. Fransz, J. WennekesEden, M.S. Ramanna, A. van Kammen, P. Zabel, and J. Hans de Jong. 1998. FISH studies reveal the molecular and chromosomal organization of individual telomere domains in tomato. Plant J. 13:507-517.

27.Sýkorová, E., J. Cartagena, M. Horáková, K. Fukui, and J. Fajkus. 2003. Characterization of telomere-subtelomere junctions in $\mathrm{Si}$ lene latifolia. Mol. Genet. Genomics 269:1320.

28.Rotková, G., M. Skleničková, M. Dvořáčková, E. Sýkorová, A.R. Leitch, and J. Fajkus. 2004. An evolutionary change in telomere sequence motif within the plant section Asparagales had significance for telomere nucleoprotein complexes. Cytogenet. Genome Res. 107:132-138.

29.Fajkus, J., R. Královics, A. Kovařík, and L. Fajkusová. 1995. The telomeric sequence is directly attached to the HRS60 subtelomeric tandem repeat in tobacco chromosomes. FEBS Lett. 364:33-35.

30.Horáková, M. and J. Fajkus. 2000. TAS49a dispersed repetitive sequence isolated from subtelomeric regions of Nicotiana tomentosiformis chromosomes. Genome 43:273-284.

31.Sýkorová, E., J. Fajkus, I. Mikako, and K. Fukui. 2001. Transition between two forms of heterochromatin at plant subtelomeres. Chromosome Res. 9:309-323.

32.Sýkorová, E., J. Cartagena, M. Horáková, K. Fukui, and J. Fajkus. 2003. Characterization of telomere-subtelomere junctions in $\mathrm{Si}$ lene latifolia. Mol. Genet. Genomics 269:1320.

33.Valárik, M., J. Bartoš, P. Kovářová, M. Kubaláková, J.H. de Jong, and J. Doležel. 2004. High-resolution FISH on superstretched flow-sorted plant chromosomes. Plant J. 37:940-950.

34.Meyne, J. and E.H. Goodwin. 1995. Direction of DNA sequences within chromatids determined using strand-specific FISH. Chromosome Res. 3:375-378.

35.Londono-Vallejo, J.A., H. Der-Sarkissian, L. Cazes, S. Bacchetti, and R.R. Reddel. 2004. Alternative lengthening of telomeres is characterized by high rates of telomeric exchange. Cancer Res. 64:2324-2327.

36.Hultdin, M., E. Gronlund, K. Norrback, E. Eriksson-Lindstrom, T. Just, and G. Roos. 1998. Telomere analysis by fluorescence in situ hybridization and flow cytometry. Nucleic Acids Res. 26:3651-3656.

37.Weiss, H. and H. Scherthan. 2002. Aloe spp.-plants with vertebrate-like telomeric sequences. Chromosome Res. 10:155-164.

38. Weiss-Schneeweiss, H., K. Ríha, C.G. Jang, J. Puizina, H. Scherthan, and D. Schweizer. 2004. Chromosome termini of the monocot plant Othocallis siberica are maintained by telomerase, which specifically synthesises vertebrate-type telomere sequences. Plant J. 37:484-493. 
39.Rabl, C. 1885. Über Zelltheilung. Morphol. Jahrb. 10:214-330.

40.Cowan, C.R., P.M. Carlton, and W.Z. Cande. 2001. The polar arrangement of telomeres in interphase and meiosis. Rabl organization and the bouquet. Plant Physiol. 125:532-538.

41.Dong, F. and J. Jiang. 1998. Non-Rabl patterns of centromere and telomere distribution in the interphase nuclei of plant cells. Chromosome Res. 6:551-558.

42.Leitch, A.R. 2000. Higher levels of organization in the interphase nucleus of cycling and differentiated cells. Microbiol. Mol. Biol. Rev. 64:138-152.

43.Bass, H.W. 2003. Telomere dynamics unique to meiotic prophase: formation and significance of the bouquet. Cell Mol. Life Sci. 60:2319-2324.

44.Zickler, D. and N. Kleckner. 1998. The leptotene-zygotene transition of meiosis. Annu. Rev. Genet. 32:619-697.

45.Carlton, P.M., C.R. Cowan, and W.Z. Cande. 2003. Directed motion of telomeres in the formation of the meiotic bouquet revealed by time course and simulation analysis. Mol. Biol. Cell 14:2832-2843.

46.Cowan, C.R. and W.Z. Cande. 2002. Meiotic telomere clustering is inhibited by colchicine but does not require cytoplasmic microtubules. J. Cell Sci. 115:3747-3756.

47.Cowan, C.R., P.M. Carlton, and W.Z. Cande. 2002. Reorganization and polarization of the meiotic bouquet-stage cell can be uncoupled from telomere clustering. J. Cell Sci. 115:3757-3766.

48.Golubovskaya, I.N., L.C. Harper, W.P. Pawlowski, D. Schichnes, and W.Z. Cande. 2002. The pam 1 gene is required for meiotic bouquet formation and efficient homologous synapsis in maize (Zea mays L.). Genetics 162:1979-1993.

49.Pawlowski, W.P., I.N. Golubovskaya, L. Timofejeva, R.B. Meeley, W.F. Sheridan, and W.Z. Cande. 2004. Coordination of meiotic recombination, pairing, and synapsis by PHS1. Science 303:89-92.

50.Bass, H.W., S.J. Bordoli, and E.M. Foss. 2003. The desynaptic (dy) and desynaptic1 (dsy1) mutations in maize (Zea mays L) cause distinct telomere-misplacement phenotypes during meiotic prophase. J. Exp. Bot. 54:3946.

51.Rossetti, L., S. Cacchione, M. Fua, and M. Savino. 1998. Nucleosome assembly on telomeric sequences. Biochemistry 37:67276737.

52.Fajkus, J. and E.N. Trifonov. 2001. Columnar packing of telomeric nucleosomes. Biochem. Biophys. Res. Commun. 280:961-963.

53.Vershinin, A.V. and J.S. Heslop-Harrison. 1998. Comparative analysis of the nucleosomal structure of rye, wheat and their relatives. Plant Mol. Biol. 36:149-161.

54. ̌̌íha, K., T.D. McKnight, J. Fajkus, B. Vyskot, and D.E. Shippen. 2000. Analysis of the G-overhang structures on plant telomeres: evidence for two distinct telomere architectures. Plant J. 23:633-641.

55.Cesare, A.J., N. Quinney, S. Willcox, D. Subramanian, and J.D. Griffith. 2003. Telomere looping in P. sativum (common garden pea). Plant J. 36:271-279.

56.Dionne, I. and R.J. Wellinger. 1996. Cell cycle-regulated generation of single-stranded Grich DNA in the absence of telomerase. Proc. Natl. Acad. Sci. USA 93:13902-13907.

57.Makarov, V.L., Y. Hirose, and J.P. Langmore. 1997. Long $\mathrm{G}$ tails at both ends of human chromosomes suggest a $\mathrm{C}$ strand degradation mechanism for telomere shortening. Cell 88:657-666.

58.Heacock, M., E. Spangler, K. Říha, J. Puizina, and D.E. Shippen. 2004. Molecular analysis of telomere fusions in Arabidopsis: multiple pathways for chromosome end-joining. EMBO J. 23:2304-2313.

59.Baird, D.M., J. Rowson, D. Wynford-Thomas, and D. Kipling. 2003. Extensive allelic variation and ultrashort telomeres in senescent human cells. Nat. Genet. 33:203-207.

60.Fajkus, J., A. Kovař́k, and R. Královics. 1996. Telomerase activity in plant cells. FEBS Lett. 391:307-309.

61.Heller, K., A. Kilian, M.A. Piatyszek, and A. Kleinhofs. 1996. Telomerase activity in plant extracts. Mol. Gen. Genet. 252:342-345.

62.Fitzgerald, M.S., T.D. McKnight, and D.E. Shippen. 1996. Characterization and developmental patterns of telomerase expression in plants. Proc. Natl. Acad. Sci. USA 93:1442214427.

63.Kim, N.W., M.A. Piatyszek, K.R. Prowse, C.B. Harley, M.D. West, P.L. Ho, G.M. Coviello, W.E. Wright, et al. 1994. Specific association of human telomerase activity with immortal cells and cancer. Science 266:20112015.

64.Fajkus, J., J. Fulnečková, M. Hulánová, K. Berková, K. Říha, and R. Matyášek. 1998. Plant cells express telomerase activity upon transfer to callus culture, without extensively changing telomere lengths. Mol. Gen. Genet. 260:470-474.

65. ̌́íha, K., J. Fajkus, J. Široký, and B. Vyskot. 1998. Developmental control of telomere lengths and telomerase activity in plants. Plant Cell 10:1691-1698.

66.Oguchi, K., H. Liu, K. Tamura, and H. Takahashi. 1999. Molecular cloning and characterization of AtTERT, a telomerase reverse transcriptase homolog in Arabidopsis thaliana. FEBS Lett. 457:465-469.

67.Fitzgerald, M.S., K. Ř́íha, F. Gao, S. Ren, T.D. McKnight, and D.E. Shippen. 1999. Disruption of the telomerase catalytic subunit gene from Arabidopsis inactivates telomerase and leads to a slow loss of telomeric DNA. Proc. Natl. Acad. Sci. USA 96:14813-14818.

68. ̌̌́ha, K., T.D. McKnight, L.R. Griffing, and D.E. Shippen. 2001. Living with genome instability: plant responses to telomere dysfunction. Science 291:1797-1800.

69.Heller-Uszynska, K., W. Schnippenkoetter, and A. Kilian. 2002. Cloning and characterization of rice (Oryza sativa L) telomerase reverse transcriptase, which reveals complex splicing patterns. Plant J. 31:75-86.

70.Ulaner, G.A., J.F. Hu, T.H. Vu, H. Oruganti, L.C. Giudice, and A.R. Hoffman. 2000. Regulation of telomerase by alternate splicing of human telomerase reverse transcriptase (hTERT) in normal and neoplastic ovary, endometrium and myometrium. Int. J. Cancer
85:330-335.

71.Colgin, L.M., C. Wilkinson, A. Englezou, A. Kilian, M.O. Robinson, and R.R. Reddel. 2000. The hTERTalpha splice variant is a dominant negative inhibitor of telomerase activity. Neoplasia 2:426-432.

72.Tamura, K., H. Liu, and H. Takahashi. 1999. Auxin induction of cell cycle regulated activity of tobacco telomerase. J. Biol. Chem. 274:20997-21002.

73.Yang, S.W., E. Jin, I.K. Chung, and W.T. Kim. 2002. Cell cycle-dependent regulation of telomerase activity by auxin, abscisic acid and protein phosphorylation in tobacco BY-2 suspension culture cells. Plant J. 29:617-626.

74.Fajkus, J., M. Šimíčková, and J. Maláska. 2002. Tiptoeing to chromosome tips: facts, promises and perils of today's human telomere biology. Phil. Trans. R. Soc. Lond. Ser B-Biol. Sci. 357:545-562.

75.Fitzgerald, M.S., E.V. Shakirov, E.E. Hood, T.D. McKnight, and D.E. Shippen. 2001 Different modes of de novo telomere formation by plant telomerases. Plant J. 26:77-87.

76.Fojtová, M., J. Fulnečková, J. Fajkus, and A. Kovařík. 2002. Recovery of tobacco cells from cadmium stress is accompanied by DNA repair and increased telomerase activity. J. Exp. Bot. 53:2151-2158.

77.Bundock, P., H. van Attikum, and P. Hooykaas. 2002. Increased telomere length and hypersensitivity to DNA damaging agents in an Arabidopsis KU70 mutant. Nucleic Acids Res. 30:3395-3400.

78. ̌́íha, K., J.M. Watson, J. Parkey, and D.E. Shippen. 2002. Telomere length deregulation and enhanced sensitivity to genotoxic stress in Arabidopsis mutants deficient in Ku70. EMBO J. 21:2819-2826.

79.Gallego, M.E., N. Jalut, and C.I. White. 2003. Telomerase dependence of telomere lengthening in Ku80 mutant Arabidopsis. Plant Cell 15:782-789.

80.Bundock, P. and P. Hooykaas. 2002. Severe developmental defects, hypersensitivity to DNA-damaging agents, and lengthened telomeres in Arabidopsis MRE11 mutants. Plant Cell 14:2451-2462.

81.Gallego, M.E. and C.I. White. 2001. RAD50 function is essential for telomere maintenance in Arabidopsis. Proc. Natl. Acad. Sci. USA 98:1711-1716.

82.Fajkus, J. and U. Zentgraf. 2002. Structure and maintenance of chromosome ends in plants, p. 314-331. In G. Krupp and R. Parwaresch (Eds.), Telomerases, Telomeres and Cancer. Landes Bioscience and Kluwer Academic, New York.

83. Kíha, K. and D.E. Shippen. 2003. Telomere structure, function and maintenance in Arabidopsis. Chromosome Res. 11:263-275.

84.Fulnečková, J. and J. Fajkus. 2000. Inhibition of plant telomerase by telomere-binding proteins from nuclei of telomerase-negative tissues. FEBS Lett. 467:305-310.

85.Kwon, C. and I.K. Chung. 2004. Interaction of an Arabidopsis RNA-binding protein with plant single-stranded telomeric DNA modulates telomerase activity. J. Biol. Chem. 279:12812-12818

86.Hirata, Y., C. Suzuki, and S. Sakai. 2004 Characterization and gene cloning of telo- 
mere-binding protein from tobacco BY-2 cells. Plant Physiol. Biochem. 42:7-14.

87.Loayza, D. and T. De Lange. 2003. POT1 as a terminal transducer of TRF1 telomere length control. Nature 424:1013-1018.

88.Colgin, L.M., K. Baran, P. Baumann, T.R. Cech, and R.R. Reddel. 2003. Human POT1 facilitates telomere elongation by telomerase. Curr. Biol. 13:942-946.

89.Liu, D., A. Safari, M.S. O'Connor, D.W. Chan, A. Laegeler, J. Qin, and Z. Songyang. 2004. PTOP interacts with POT1 and regulates its localization to telomeres. Nat. Cell Biol. 6:673-680.

90.Yang, S.W., D.H. Kim, J.J. Lee, Y.J. Chun, J.H. Lee, Y.J. Kim, I.K. Chung, and W.T. Kim. 2003. Expression of the telomeric repeat binding factor gene NgTRF1 is closely coordinated with the cell division program in tobacco BY-2 suspension culture cells. J. Biol. Chem. 278:21395-21407.

91.Schrumpfova, P., M. Kuchar, G. Mikova, L. Skrisovska, T. Kubicarova, and J. Fajkus. 2004. Characterization of two Arabidopsis thaliana myb-like proteins showing affinity to telomeric DNA sequence. Genome 47:316324.

92.Baumann, P., E. Podell, and T.R. Cech. 2002. Human Pot1 (protection of telomeres) protein: cytolocalization, gene structure, and alternative splicing. Mol. Cell Biol. 22:80798087.

93.Makarov, V.L., S. Lejnine, J. Bedoyan, and J.P. Langmore. 1993. Nucleosomal organization of telomere-specific chromatin in rat. Cell 73:775-787.

94.Tommerup, H., A. Dousmanis, and T. de Lange. 1994. Unusual chromatin in human telomeres. Mol. Cell Biol. 14:5777-5785.

95.Ascenzi, R. and J.S. Gantt. 1999. Subnuclear distribution of the entire complement of linker histone variants in Arabidopsis thaliana. Chromosoma 108:345-355.

96.Sharma, G.G., K.K. Hwang, R.K. Pandita, A. Gupta, S. Dhar, J. Parenteau, M. Agarwal, H.J. Worman, et al. 2003. Human heterochromatin protein 1 isoforms HP1(Hsalpha) and HP1(Hsbeta) interfere with hTERT-telomere interactions and correlate with changes in cell growth and response to ionizing radiation. Mol. Cell Biol. 23:8363-8376.

97.Sadaie, M., T. Naito, and F. Ishikawa. 2003. Stable inheritance of telomere chromatin structure and function in the absence of telomeric repeats. Genes Dev. 17:2271-2282.

\section{Address correspondence to:}

Jiří Fajkus,

Institute of Biophysics ASCR

Královopolská 135CZ-61265

Brno, Czech Republic

e-mail:fajkus@ibp.cz 